\title{
Correction to: Central IGF1 improves glucose tolerance and insulin sensitivity in mice
}

\author{
Hao Hong, Zhen-Zhong Cui, Lu Zhu OD, Shu-Ping Fu, Mario Rossi, Ying-Hong Cui and Bing-Mei Zhu
}

\section{Correction to: Nutrition and Diabetes \\ https://doi.org/10.1038/s41387-017-0002-0 \\ published online 19 December 2017}

The original version of this article unfortunately contained a mistake in the affiliations. The correct affiliations are: Hao Hong 1 Key Laboratory of Acupuncture and Medicine Research of Ministry of Education, Nanjing University of Chinese Medicine, Nanjing, Jiangsu 210023, China. Zhen-Zhong Cui 2 Molecular Signaling Section, Laboratory of Bioorganic Chemistry, National Institute of Diabetes and Digestive and Kidney Diseases, Bethesda, MD 20892, USA.

Lu Zhu 2 Molecular Signaling Section, Laboratory of Bioorganic Chemistry, National Institute of Diabetes and Digestive and Kidney Diseases, Bethesda, MD 20892, USA.
Shu-Ping Fu 1 Key Laboratory of Acupuncture and Medicine Research of Ministry of Education, Nanjing University of Chinese Medicine, Nanjing, Jiangsu 210023, China.

Mario Rossi 2 Molecular Signaling Section, Laboratory of Bioorganic Chemistry, National Institute of Diabetes and Digestive and Kidney Diseases, Bethesda, MD 20892, USA. Ying-Hong Cui 2 Molecular Signaling Section, Laboratory of Bioorganic Chemistry, National Institute of Diabetes and Digestive and Kidney Diseases, Bethesda, MD 20892, USA.

Bing-Mei Zhu 3 Regenerative Medicine Research Center, West China Hospital, Sichuan University, Keyuan Road 4, Gaopeng Street, Chengdu, Sichuan 610041, China.

Published online: 19 February 2021 